\title{
New Construction Weighted $(h, q)$-Genocchi Numbers and Polynomials Related to Zeta Type Functions
}

\author{
Serkan Araci, ${ }^{1}$ Jong Jin Seo, ${ }^{2}$ and Dilek Erdal ${ }^{1}$ \\ ${ }^{1}$ Department of Mathematics, Faculty of Arts and Science, University of Gaziantep, \\ 27310 Gaziantep, Turkey \\ 2 Department of Applied Mathematics, Pukyong National University, \\ Busan 608-737, Republic of Korea
}

Correspondence should be addressed to Jong Jin Seo, seo2011@pknu.ac.kr

Received 31 March 2011; Revised 17 June 2011; Accepted 11 July 2011

Academic Editor: Guang Zhang

Copyright (c) 2011 Serkan Araci et al. This is an open access article distributed under the Creative Commons Attribution License, which permits unrestricted use, distribution, and reproduction in any medium, provided the original work is properly cited.

The fundamental aim of this paper is to construct $(h, q)$-Genocchi numbers and polynomials with weight $\alpha$. We shall obtain some interesting relations by using $p$-adic $q$-integral on $\mathbb{Z}_{p}$ in the sense of fermionic. Also, we shall derive the $(h, q)$-extensions of zeta type functions with weight $\alpha$ from the Mellin transformation of this generating function which interpolates the $(h, q)$-Genocchi numbers and polynomials with weight $\alpha$ at negative integers.

\section{Introduction, Definitions, and Notations}

Let $p$ be a fixed odd prime number. Throughout this paper we use the following notations. $\mathbb{Z}_{p}$ denotes the ring of $p$-adic rational integers, $\mathbb{Q}$ denotes the field of rational numbers, $\mathbb{Q}_{p}$ denotes the field of $p$-adic rational numbers, and $\mathbb{C}_{p}$ denotes the completion of algebraic closure of $\mathbb{Q}_{p}$. Let $\mathbb{N}$ be the set of natural numbers and $\mathbb{N}^{*}=\mathbb{N} \cup\{0\}$. The $p$-adic absolute value is defined by $|p|_{p}=1 / p$. In this paper, we assume $|q-1|_{p}<1$ as an indeterminate. In [1-3], Kim defined the fermionic $p$-adic $q$-integral on $\mathbb{Z}_{p}$ as follows:

$$
I_{-q}(f)=\int_{\mathbb{Z}_{p}} f(x) d \mu_{-q}(x)=\lim _{N \rightarrow \infty} \frac{1}{\left[p^{N}\right]_{-q}} \sum_{x=0}^{p^{N}-1} f(x)(-q)^{x}
$$


$[x]_{q}$ is a $q$-extension of $x$ which is defined by

$$
[x]_{q}=\frac{1-q^{x}}{1-q}
$$

see $[1-15]$.

Note that $\lim _{q \rightarrow 1}[x]_{q}=x$.

Let $f_{n}(x)=f(x+n)$. By the definition (1.1) we easily get

$$
\begin{aligned}
-q I_{-q}\left(f_{1}\right) & =\lim _{N \rightarrow \infty} \frac{1}{\left[p^{N}\right]_{-q}} \sum_{x=0}^{p^{N}-1} f(x+1)(-q)^{x+1} \\
& =\lim _{N \rightarrow \infty} \frac{1}{\left[p^{N}\right]_{-q}} \sum_{x=0}^{p^{N}-1} f(x)(-q)^{x}-(1+q) \lim _{N \rightarrow \infty} \frac{f\left(p^{N}\right) q^{p^{N}}+f(0)}{1+q^{p^{N}}} \\
& =I_{-q}(f)-[2]_{q} f(0) .
\end{aligned}
$$

Continuing this process, we obtain easily the relation

$$
q^{n} I_{-q}\left(f_{n}\right)+(-1)^{n-1} I_{-q}(f)=[2]_{q} \sum_{l=0}^{n-1}(-1)^{n-l-1} q^{l} f(l),
$$

$(h, q)$-Genocchi numbers are defined as follows:

$$
G_{0, q}^{(h)}=0, \quad q^{h-2}\left(q G_{q}^{(h)}+1\right)^{n}+G_{n, q}^{(h)}= \begin{cases}{[2]_{q},} & \text { if } n=1, \\ 0, & \text { if } n>1,\end{cases}
$$

with the usual convention about replacing $\left(G_{q}^{(h)}\right)^{n}$ by $G_{n, q}^{(h)}$ (see [6]).

In this paper, we constructed $(h, q)$-Genocchi numbers and polynomials with weight $\alpha$. By using fermionic $p$-adic $q$-integral equations on $\mathbb{Z}_{p}$, we investigated some interesting identities and relations on the $(h, q)$-Genocchi numbers and polynomials with weight $\alpha$. Furthermore, we derive the $q$-extensions of zeta type functions with weight $\alpha$ from the Mellin transformation of this generating function which interpolates the $(h, q)$-Genocchi polynomials with weight $\alpha$.

\section{On the Weighted $(h, q)$-Genocchi Numbers and Polynomials}

In this section, by using fermionic $p$-adic $q$-integral equations on $\mathbb{Z}_{p}$, some interesting identities and relation on the $(h, q)$-Genocchi numbers and polynomials with weight $\alpha$ are shown.

Definition 2.1. Let $\alpha, n \in \mathbb{N}^{*}$ and $h \in \mathbb{N}$. Then the $(h, q)$-Genocchi numbers with weight $\alpha$ defined by as follows:

$$
\frac{\tilde{G}_{n+1, q}^{(\alpha, h)}}{n+1}=[2]_{q} \sum_{m=0}^{\infty}(-1)^{m} q^{m h}[m]_{q^{\alpha}}^{n}
$$


If we take $h=1$ to (2.1), then we have, $\widetilde{G}_{n+1, q}^{(\alpha, 1)}=\widetilde{G}_{n+1, q}^{(\alpha)}$ (see [5]).

From (2.1), we obtain

$$
\begin{aligned}
\frac{\tilde{G}_{n+1, q}^{(\alpha, h)}}{n+1} & =\frac{[2]_{q}}{\left(1-q^{\alpha}\right)^{n}} \sum_{m=0}^{\infty}(-1)^{m} q^{m h}\left(1-q^{m \alpha}\right)^{n} \\
& =\frac{[2]_{q}}{\left(1-q^{\alpha}\right)^{n}}\left[\sum_{m=0}^{\infty}(-1)^{m} q^{m h} \sum_{l=0}^{n}\left(\begin{array}{l}
n \\
l
\end{array}\right)(-1)^{l}\left(q^{m \alpha}\right)^{l}\right] \\
& =\frac{[2]_{q}}{\left(1-q^{\alpha}\right)^{n}} \sum_{l=0}^{n}\left(\begin{array}{l}
n \\
l
\end{array}\right)(-1)^{l} \sum_{m=0}^{\infty}(-1)^{m} q^{m \alpha l+m h} \\
& =\frac{[2]_{q}}{\left(1-q^{\alpha}\right)^{n}} \sum_{l=0}^{n}\left(\begin{array}{l}
n \\
l
\end{array}\right)(-1)^{l} \frac{1}{1+q^{\alpha l+h}}
\end{aligned}
$$

Therefore, we obtain the following theorem.

Theorem 2.2. For $\alpha, n \in \mathbb{N}^{*}$ and $h \in \mathbb{N}$. Then

$$
\frac{\tilde{G}_{n+1, q}^{(\alpha, h)}}{n+1}=\frac{[2]_{q}}{\left(1-q^{\alpha}\right)^{n}} \sum_{l=0}^{n}\left(\begin{array}{l}
n \\
l
\end{array}\right)(-1)^{l} \frac{1}{1+q^{\alpha l+h}} .
$$

In (1.1), one takes $f(x)=q^{(h-1) x}[x]_{q^{\alpha}}^{n}$

$$
\begin{aligned}
\int_{\mathbb{Z}_{p}} q^{(h-1) x}[x]_{q^{\alpha}}^{n} d \mu_{-q}(x) & =\frac{1}{\left(1-q^{\alpha}\right)^{n}} \sum_{l=0}^{n}\left(\begin{array}{l}
n \\
l
\end{array}\right)(-1)^{l} \int_{\mathbb{Z}_{p}} q^{x(\alpha l+h-1)} d \mu_{-q}(x) \\
& =\frac{1}{\left(1-q^{\alpha}\right)^{n}} \sum_{l=0}^{n}\left(\begin{array}{l}
n \\
l
\end{array}\right)(-1)^{l} \lim _{N \rightarrow \infty} \frac{1}{\left[p^{N}\right]_{-q}} \sum_{x=0}^{p^{N}-1}\left(-q^{\alpha l+h}\right)^{x} \\
& =\frac{1}{\left(1-q^{\alpha}\right)^{n}} \sum_{l=0}^{n}\left(\begin{array}{l}
n \\
l
\end{array}\right)(-1)^{l} \frac{(1+q)}{1+q^{\alpha l+h}} \lim _{N \rightarrow \infty} \frac{1+\left(q^{\alpha l+h}\right)^{p^{N}}}{1+q^{p^{N}}} \\
& =\frac{[2]_{q}}{\left(1-q^{\alpha}\right)^{n}} \sum_{l=0}^{n}\left(\begin{array}{l}
n \\
l
\end{array}\right)(-1)^{l} \frac{1}{1+q^{\alpha l+h}} \\
& =\frac{\tilde{G}_{n+1, q}^{(\alpha, h)}}{n+1} .
\end{aligned}
$$

From [12], we obtain $(h, q)$-Genocchi numbers with weight $\alpha$ witt's type formula as follows. 
Theorem 2.3. For $\alpha, n \in \mathbb{N}^{*}$ and $h \in \mathbb{N}$. Then

$$
\frac{\tilde{G}_{n+1, q}^{(\alpha, h)}}{n+1}=\int_{\mathbb{Z}_{p}} q^{(h-1) x}[x]_{q^{\alpha}}^{n} d \mu_{-q}(x) .
$$

From (2.1), one easily gets

$$
\int_{\mathbb{Z}_{p}} q^{(h-1) x} e^{t[x]_{q^{\alpha}}} d \mu_{-q}(x)=t[2]_{q} \sum_{m=0}^{\infty}(-1)^{m} q^{m h} e^{t[m]_{q^{\alpha}}}
$$

By (2.6), one has

$$
\sum_{n=0}^{\infty} \widetilde{G}_{n, q}^{(\alpha, h)} \frac{t^{n}}{n !}=t[2]_{q} \sum_{m=0}^{\infty}(-1)^{m} q^{m h} e^{t[m]_{q^{\alpha}}}
$$

Therefore, we obtain the following corollary.

Corollary 2.4. If $\widetilde{G}_{0, q}^{(\alpha, h)}=0$. Let $D_{q}^{(\alpha, h)}(t)=\sum_{n=0}^{\infty} \widetilde{G}_{n, q}^{(\alpha, h)}\left(t^{n} / n !\right)$. Then

$$
D_{q}^{(\alpha, h)}(t)=t[2]_{q} \sum_{m=0}^{\infty}(-1)^{m} q^{m h} e^{t[m]_{q^{\alpha}}}
$$

Now, one considers the $(h, q)$-Genocchi polynomials with weight $\alpha$ as follows:

$$
\frac{\tilde{G}_{n+1, q}^{(\alpha, h)}(x)}{n+1}=\int_{\mathbb{Z}_{p}} q^{(h-1) y}[x+y]_{q^{\alpha}}^{n} d \mu_{-q}(y), \quad n \in \mathbb{N}, \alpha \in \mathbb{N}^{*} .
$$

From (2.9), one sees that

$$
\frac{\tilde{G}_{n+1, q}^{(\alpha, h)}(x)}{n+1}=\frac{[2]_{q}}{\left(1-q^{\alpha}\right)^{n}} \sum_{l=0}^{n}\left(\begin{array}{l}
n \\
l
\end{array}\right)(-1)^{l} q^{\alpha l x} \frac{1}{1+q^{\alpha l+h}}=[2]_{q} \sum_{m=0}^{\infty}(-1)^{m} q^{m h}[m+x]_{q^{\alpha}}^{n} .
$$

Let $D_{q}^{(\alpha, h)}(t, x)=\sum_{n=0}^{\infty} \widetilde{G}_{n, q}^{(\alpha, h)}(x)\left(t^{n} / n !\right)$. Then, one has

$$
D_{q}^{(\alpha, h)}(t, x)=t[2]_{q} \sum_{m=0}^{\infty}(-1)^{m} q^{m h} e^{t[m+x]_{q^{\alpha}}}=\sum_{n=0}^{\infty} \widetilde{G}_{n, q}^{(\alpha, h)}(x) \frac{t^{n}}{n !} .
$$

By (1.4), one sees that

$$
q^{h n} \frac{\tilde{G}_{m+1, q}^{(\alpha, h)}(n)}{m+1}+(-1)^{n-1} \frac{\tilde{G}_{m+1, q}^{(\alpha, h)}}{m+1}=[2]_{q} \sum_{l=0}^{n-1}(-1)^{n-l-1} q^{h l}[l]_{q^{\alpha}}^{m} .
$$


Therefore, we obtain the following theorem.

Theorem 2.5. For $m, h \in \mathbb{N}$, and $\alpha, n \in \mathbb{N}^{*}$, one has

$$
q^{h n} \frac{\tilde{G}_{m+1, q}^{(\alpha, h)}(n)}{m+1}+(-1)^{n-1} \frac{\tilde{G}_{m+1, q}^{(\alpha, h)}}{m+1}=[2]_{q} \sum_{l=0}^{n-1}(-1)^{n-l-1} q^{h l}[l]_{q^{\alpha}}^{m}
$$

In (1.3), it is known that

$$
q I_{-q}\left(f_{1}\right)+I_{-q}(f)=[2]_{q} f(0)
$$

If we take $f(x)=q^{(h-1) x} e^{t[x]_{q^{\alpha}}}$, then one has

$$
\begin{aligned}
{[2]_{q} } & =q \int_{\mathbb{Z}_{p}} q^{(h-1)(x+1)} e^{t[x+1]_{q^{\alpha}}} d \mu_{-q}(x)+\int_{\mathbb{Z}_{p}} q^{(h-1) x} e^{t[x]_{q^{\alpha}}} d \mu_{-q}(x) \\
& =\sum_{m=0}^{\infty}\left(q^{h} \frac{\tilde{G}_{m+1, q}^{(\alpha, h)}(1)}{m+1}+\frac{\widetilde{G}_{m+1, q}^{(\alpha, h)}}{m+1}\right) \frac{t^{m}}{m !} .
\end{aligned}
$$

Therefore, by (2.15), we obtain the following theorem.

Theorem 2.6. For $\alpha \in \mathbb{N}^{*}$ and $m, h \in \mathbb{N}$, one has

$$
\tilde{G}_{0, q}^{(\alpha, h)}=0, \quad q^{h} \frac{\tilde{G}_{m+1, q}^{(\alpha, h)}(1)}{m+1}+\frac{\tilde{G}_{m+1, q}^{(\alpha, h)}}{m+1}= \begin{cases}{[2]_{q},} & \text { if } m=0 \\ 0, & \text { if } m \neq 0\end{cases}
$$

From (2.9), one can easily derive the following:

$$
\begin{aligned}
\int_{\mathbb{Z}_{p}} q^{(h-1) y}[x+y]_{q^{\alpha}}^{n} d \mu_{-q}(y) & =\frac{[d]_{q^{\alpha}}^{n}}{[d]_{-q}} \sum_{a=0}^{d-1}(-1)^{a} q^{h a} \int_{\mathbb{Z}_{p}} q^{d y(h-1)}\left[\frac{x+a}{d}+y\right]_{q^{d \alpha}}^{n} d \mu_{(-q)^{d}}(y) \\
& =\frac{[d]_{q^{\alpha}}^{n}}{[d]_{-q}} \sum_{a=0}^{d-1}(-1)^{a} q^{h a} \frac{\widetilde{G}_{n+1, q^{d}}^{(\alpha, h)}((x+a) / d)}{n+1}
\end{aligned}
$$

Therefore, by (2.17), we obtain the following theorem.

Theorem 2.7. For $d \equiv 1(\bmod 2), n \in \mathbb{N}^{*}$ and $\alpha, h \in \mathbb{N}$

$$
\tilde{G}_{n+1, q}^{(\alpha, h)}(x)=\frac{[d]_{q^{\alpha}}^{n}}{[d]_{-q}} \sum_{a=0}^{d-1}(-1)^{a} q^{h a} \tilde{G}_{n+1, q^{d}}^{(\alpha, h)}\left(\frac{x+a}{d}\right) .
$$




\section{Interpolation Function of the Polynomials $\widetilde{G}_{n, q}^{(\alpha, h)}(x)$}

In this section, we give interpolation function of the generating functions of $(h, q)$-Genocchi polynomials with weight $\alpha$. For $s \in \mathbb{C}$ and $h \in \mathbb{N}$, by applying the Mellin transformation to (2.11), we obtain

$$
\Im_{q}^{(\alpha, h)}(s, x)=\frac{1}{\Gamma(s)} \int_{0}^{\infty} t^{s-2}\left(-D_{q}^{(\alpha, h)}(-t, x)\right) d t=[2]_{q} \sum_{m=0}^{\infty}(-1)^{m} q^{m h} \frac{1}{\Gamma(s)} \int_{0}^{\infty} t^{s-1} e^{-t[m+x]_{q^{\alpha}}} d t
$$

so we have

$$
\mathfrak{I}_{q}^{(\alpha, h)}(s, x)=[2]_{q} \sum_{m=0}^{\infty} \frac{(-1)^{m} q^{m h}}{[m+x]_{q^{\alpha}}^{s}} .
$$

We define $q$-extension zeta type function as follows.

Theorem 3.1. For $s \in \mathbb{C}, h \in \mathbb{N}$, and $\alpha \in \mathbb{N}^{*}$. One has

$$
\mathfrak{I}_{q}^{(\alpha, h)}(s, x)=[2]_{q} \sum_{m=0}^{\infty} \frac{(-1)^{m} q^{m h}}{[m+x]_{q^{\alpha}}^{s}} .
$$

$\mathfrak{I}_{q}^{(\alpha, h)}(s, x)$ can be continued analytically to an entire function.

By subsituting $s=-n$ into (3.3) one easily gets

$$
\mathfrak{I}_{q}^{(\alpha, h)}(-n, x)=\frac{\widetilde{G}_{n+1, q}^{(\alpha, h)}(x)}{n+1} .
$$

We obtain the following theorem.

Theorem 3.2. For $h \in \mathbb{N}$ and $q, s \in \mathbb{C},|q|<1$. Then one defines

$$
\mathfrak{I}_{q}^{(\alpha, h)}(-n, x)=\frac{\tilde{G}_{n+1, q}^{(\alpha, h)}(x)}{n+1} .
$$

\section{References}

[1] T. Kim, " $q$-Euler numbers and polynomials associated with $p$-adic $q$-integrals," Journal of Nonlinear Mathematical Physics, vol. 14, no. 1, pp. 15-27, 2007.

[2] T. Kim, "New approach to q-Euler polynomials of higher order," Russian Journal of Mathematical Physics, vol. 17, no. 2, pp. 218-225, 2010.

[3] T. Kim, "Some identities on the $q$-Euler polynomials of higher order and $q$-Stirling numbers by the fermionic $p$-adic integral on $\mathbb{Z}_{p}, "$ Russian Journal of Mathematical Physics, vol. 16, no. 4, pp. 484-491, 2009.

[4] M. Acikgoz and Y. Simsek, "A new generating function of $q$-Bernstein-type polynomials and their interpolation function," Abstract and Applied Analysis, vol. 2010, Article ID 769095, 12 pages, 2010. 
[5] S. Araci, D. Erdal, and J. J. Seo, "A Study on the weighted $q$-Genocchi Numbers and Polynomials Their Interpolation function," submitted.

[6] S. Araci, J. J. Seo, and D. Erdal, "Different Approach On The $(h, q)$ Genocchi Numbers and Polynomials Associated with $q$-Bernstein Polynomials," submitted.

[7] T. Kim, "A new approach to q-Zeta function," Advanced Studies in Contemporary Mathematics, vol. 11, no. 2, pp. 157-162, 2005.

[8] T. Kim, "On the q-extension of Euler and Genocchi numbers," Journal of Mathematical Analysis and Applications, vol. 326, no. 2, pp. 1458-1465, 2007.

[9] T. Kim, "On the multiple $q$-Genocchi and Euler numbers," Russian Journal of Mathematical Physics, vol. 15, no. 4, pp. 481-486, 2008.

[10] T. Kim, "On the weighted $q$-Bernoulli numbers and polynomials," Advanced Studies in Contemporary Mathematics, vol. 21, no. 2, pp. 207-215, 2011.

[11] T. Kim, "A note on the $q$-Genocchi numbers and polynomials," Journal of Inequalities and Applications, vol. 2007, Article ID 71452, 8 pages, 2007.

[12] T. Kim, “q-Volkenborn integration," Russian Journal of Mathematical Physics, vol. 9, no. 3, pp. 288-299, 2002.

[13] T. Kim, "An invariant $p$-adic $q$-integral on $\mathbb{Z}_{p}$, " Applied Mathematics Letters, vol. 21, no. 2, pp. 105-108, 2008.

[14] T. Kim, "A note on the q-genocchi numbers and polynomials," Journal of Inequalities and Applications, vol. 2007, Article ID 71452, 8 pages, 2007.

[15] T. Kim, J. Choi, Y. H. Kim, and L. C. Jang, “On $p$-Adic analogue of $q$-Bernstein polynomials and related integrals," Discrete Dynamics in Nature and Society, vol. 2010, Article ID 179430, 9 pages, 2010. 


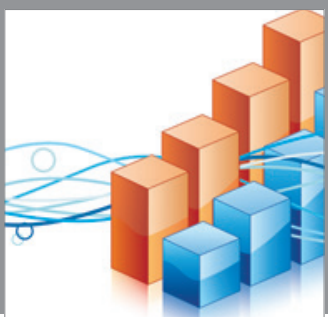

Advances in

Operations Research

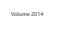

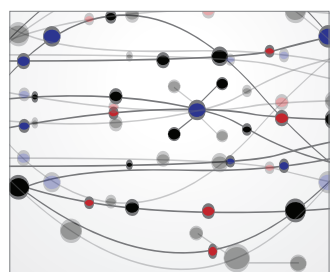

\section{The Scientific} World Journal
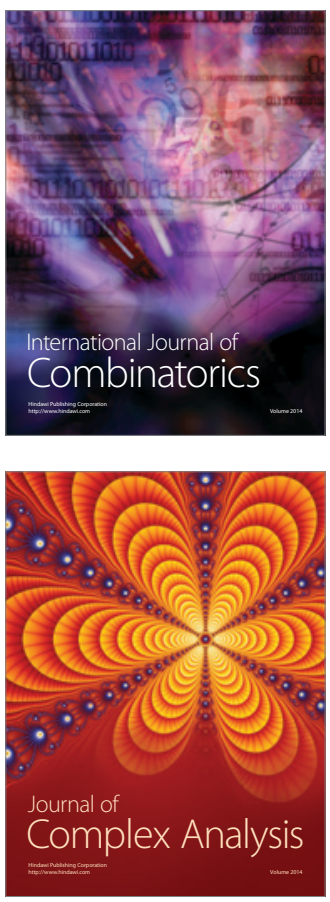

International Journal of

Mathematics and

Mathematical

Sciences
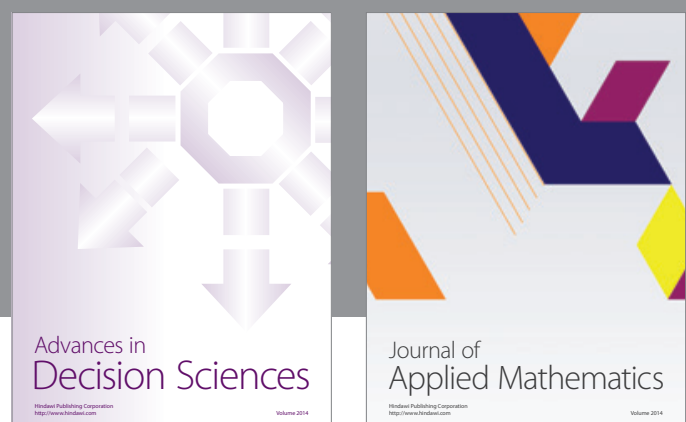

Journal of

Applied Mathematics
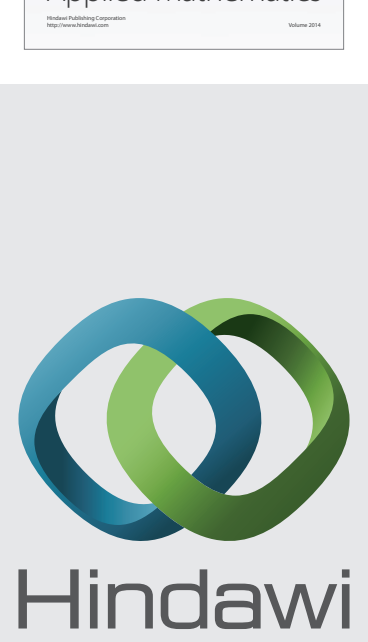

Submit your manuscripts at http://www.hindawi.com
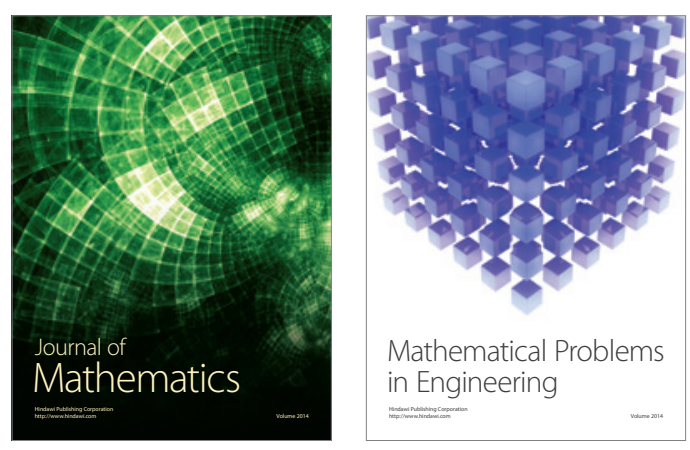

Mathematical Problems in Engineering
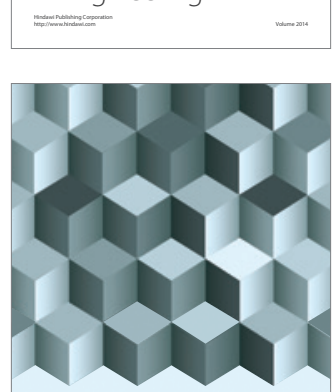

Journal of

Function Spaces
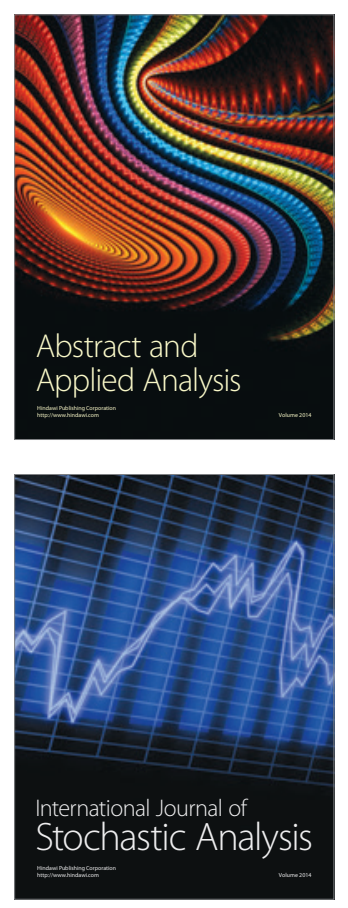

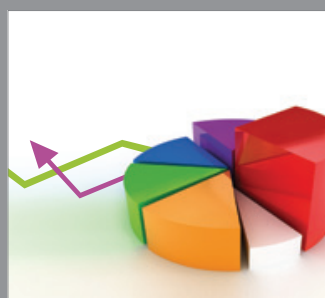

ournal of

Probability and Statistics

Promensencen
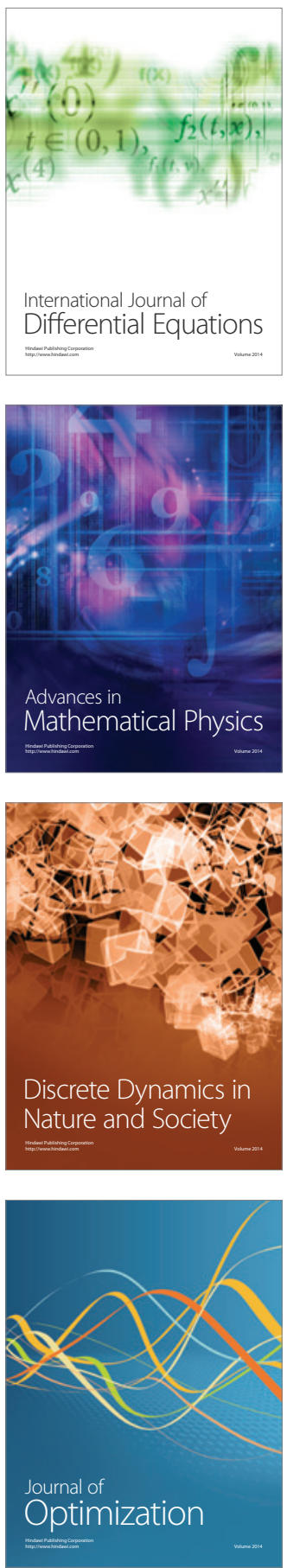